\title{
SENSITIVITY ANALYSIS FOR VARIATIONAL INCLUSIONS BY WIENER-HOPF EQUATION TECHNIQUES
}

\author{
ABDELLATIF MOUDAFI \\ Université des Antilles et de la Guyane \\ Mathématiques \\ 97159 Pointe-à-Pitre, Guadeloupe \\ MUHAMMAD ASLAM NOOR \\ Dalhousie University \\ Mathematics and Statistics \\ Halifax, Nova Scotia \\ Canada B3H $3 \mathrm{~J} 5$
}

(Received July, 1997; Revised May, 1998)

In this paper, we extend the sensitivity analysis framework developed recently for variational inequalities by Noor and Yen to variational inclusions relying on Wiener-Hopf equation techniques. We prove the continuity and the Lipschitz continuity of the locally unique solution to parametric variational inclusions without assuming differentiability of the given data.

Key words: Variational Inclusions, Wiener-Hopf Equations, Sensitivity Analysis, Resolvent Operator.

AMS subject classifications: $49 \mathrm{~J} 40,90 \mathrm{C} 33$.

\section{Introduction}

Variational inequalities theory has emerged as an interesting branch of applicable mathematics which enables us to study a large number of problems arising in economics, optimization, and operations research in a general and unified way. Numerous numerical methods are now available for finding the approximate solutions to variational inequalities and variational inclusions. Recently, much attention has been given to develop sensitivity framework for variational inequalities using quite different techniques, see for example, Dafermos [5], Tobin [21], Kyparisis [9], Robinson [18]. Some results have been obtained with special structures; see for instance, Qui-Magnanti [17], Janin-Gauvin [8], and Noor [12]. Inspired and motivated by the recent research in this field, we consider the class of variational inclusions, which includes variational inequalities, complementarity problems, convex optimization, and saddle point problems as special cases. 
Variational inclusions have potential and useful applications in optimization and economics, see [1-23]. Using Wiener-Hopf equation techniques and ideas of Dafermos [5] and Noor [12], we develop a sensitivity analysis for variational inclusions. In the process, we establish the equivalence between variational inclusions and Wiener-Hopf equations. This equivalence provides us with a new approach for studying sensitivity analysis for this kind of inclusions by relying on a fixed-point formulation of the given problem. We would like to emphasize that our approach is totally different from the techniques of Robinson [18] based on the Wiener-Hopf equations coupled with implicit-function theorem, as well as those of Pang-Ralph [16], which use the degree theory for studying the piecewise smoothness and local invertibility of the parametric normal (Wiener-Hopf) equations.

\section{Preliminaries}

Let $X$ be a real Hilbert space and $\|\cdot\|$ the norm generated by the scalar product $\langle\cdot, \cdot\rangle$. Let $A, g$ be nonlinear operators, and $B$ a maximal monotone operator. Consider the problem:

$$
\text { find } x \in X \text { such that } 0 \in A x+B(g(x)) \text {, }
$$

which is called the general variational inclusion and generalized the concept of variational inequalities [13-15].

Related to this problem, we consider the equation:

$$
\text { find } z \in X \text { such that } A g^{-1} J_{\mu}^{B}(z)+B_{\mu} z=0 \text {. }
$$

where $\mu>0$ is a real constant, $J_{\mu}^{B}:=(I+\mu B)^{-1}$ and $B_{\mu}:=\frac{1}{\mu}\left(I-J_{\mu}^{B}\right)$ are the resolvent and the Yosida approximate associated with $B$, respectively, and $I$ stands for the identity on $X$ and $g$ is injective. The equations of the type (2.2) are called the generalized Wiener-Hopf equations or the resolvent equations. For the applications and formulations of the resolvent equations, see Noor [13-15].

We recall that the resolvent mapping is nonexpansive, i.e.,

$$
\left\|J_{\mu}^{B} x-J_{\mu}^{B} y\right\| \leq\|x-y\| \quad \forall x, y \in X
$$

the Yosida approximate is Lipschitz continuous with constant $\frac{1}{\mu}$ :

$$
\left\|B_{\mu} x-B_{\mu} y\right\| \leq \frac{1}{\mu}\|x-y\| \quad \forall x, y \in X,
$$

and they are related by the following formula:

$$
B_{\mu} x \in B\left(J_{\mu}^{B} x\right) \quad \forall x, y \in X .
$$

Now, we consider the parametric versions of problems (2.1) and (2.2). To formulate the problems, let $\Lambda$ be an open subset of a Hilbert space $Y$ in which $\lambda$ takes values, and $|\cdot|$ be the norm generated by its scalar product. Then the parametric version of (2.1) is given by: 


$$
\text { find } x_{\lambda} \in X \text { such that } 0 \in A\left(x_{\lambda}, \lambda\right)+B\left(g\left(x_{\lambda}, \lambda\right), \lambda\right) \text {, }
$$

where $A(\cdot, \lambda): X \times \Lambda \rightarrow X, B(\cdot, \lambda): X \times \Lambda \rightarrow X$ are given operators.

The associated parametric Wiener-Hopf equation is:

$$
\text { find } z_{\lambda} \in X ; \quad A g^{-1} J_{\mu}^{B(\cdot, \lambda)} z_{\lambda}+(B(\cdot, \lambda))_{\mu} z_{\lambda}=0 .
$$

We assume that for some $\bar{\lambda} \in \Lambda$, problem (2.3) has a unique solution $\bar{x}$. We will show that in this case, (2.4) also has a unique solution $\bar{z}$. In what follows, we are interested in knowing if (2.3) (respectively, (2.4)) has a solution, denoted $x_{\lambda}$ (respectively, $z_{\lambda}$ ), close to $\bar{x}$ (respectively, $\bar{z}$ ) when $\lambda$ is close to $\bar{\lambda}$, and how the function $x(\lambda):=x_{\lambda}$ (respectively, $z(\lambda):=z_{\lambda}$ ) behaves. In other words, we want to investigate the sensitivity of the solutions $\bar{x}$ and $\bar{z}$ with respect to change of the parameter $\lambda$.

The object of the next result is to establish the equivalence between (2.3) and (2.4).

Lemma 2.1: The parametric variational inclusion in (2.3) has a solution $x_{\lambda}$ if and only if the parametric Wiener-Hopf equation in (2.3) has a solution $z_{\lambda}$, where:

$$
g\left(x_{\lambda}, \lambda\right)=J_{\mu}^{B(\cdot, \lambda)} z_{\lambda} \text { and } z_{\lambda}=g\left(x_{\lambda}, \lambda\right)-\mu A\left(x_{\lambda}, \lambda\right)
$$

Proof: Let $x_{\lambda}$ be a solution of (2.3), i.e.

which is equivalent to

$$
-A\left(x_{\lambda}, \lambda\right) \in B\left(g\left(x_{\lambda}, \lambda\right), \lambda\right)
$$

$$
g\left(x_{\lambda}, \lambda\right)-\mu A\left(x_{\lambda}, \lambda\right) \in g\left(x_{\lambda}, \lambda\right)+\mu B\left(g\left(x_{\lambda}, \lambda\right), \lambda\right)
$$

Thus

$$
g\left(x_{\lambda}, \lambda\right)=J_{\mu}^{B(\cdot, \lambda)}\left(g\left(x_{\lambda}, \lambda\right)-\mu A\left(x_{\lambda}, \lambda\right)\right)
$$

This, combined with definition of the Yosida approximate, yields

$$
(B(\cdot, \lambda))_{\mu}\left(g\left(x_{\lambda}, \lambda\right)-\mu A\left(x_{\lambda}, \lambda\right)\right)=-A\left(x_{\lambda}, \lambda\right)
$$

that is

where

$$
A\left(x_{\lambda}, \lambda\right)+(B(\cdot, \lambda))_{\mu}\left(z_{\lambda}\right)=0
$$

$$
z_{\lambda}=g\left(x_{\lambda}, \lambda\right)-\mu A\left(x_{\lambda}, \lambda\right)
$$

Conversely, let $z_{\lambda} \in X$ be a solution of (2.4). Then

$$
A\left(x_{\lambda}, \lambda\right)+(B(\cdot, \lambda))_{\mu}\left(z_{\lambda}\right)=0 \text { with } g\left(x_{\lambda}, \lambda\right)=J_{\mu}^{B(\cdot, \lambda)} z_{\lambda}
$$

which yields that

$$
(B(\cdot, \lambda))_{\mu}\left(z_{\lambda}\right) \in B\left(x_{\lambda}, \lambda\right)
$$

This, combined with (2.3) gives:

$$
0 \in A\left(x_{\lambda}, \lambda\right)+B\left(g\left(x_{\lambda}, \lambda\right), \lambda\right) .
$$


Thus, $x_{\lambda}$ is a solution of $(2,3)$.

Remark 2.1: (i) We can give another proof based on an abstract duality principle for operators. Indeed (2.3) is equivalent to the problem

$$
\text { find } x_{\lambda} \in X ; 0 \in-g\left(x_{\lambda}, \lambda\right)+\mu A\left(x_{\lambda}, \lambda\right)+g\left(x_{\lambda}, \lambda\right)+\mu B\left(g\left(x_{\lambda}, \lambda\right), \lambda\right) \text {. }
$$

Setting $\boldsymbol{A}:=-g+\mu A$ and $\boldsymbol{B}:=(I+\mu B) \circ g$, and applying the abstract duality principle (Attouch-Théra [2]), (2.3) is equivalent to:

$$
\text { find } z_{\lambda} \in X ; 0 \in z_{\lambda}+\boldsymbol{A} \boldsymbol{B}^{-1} z_{\lambda} \text { with } z_{\lambda} \in \boldsymbol{B} x_{\lambda} \text {. }
$$

Noticing that $\boldsymbol{B}^{-1} z_{\lambda}$ is nothing but $g^{-1}\left(J_{\mu}^{B(\cdot, \lambda)} z_{\lambda}\right)$, we derive:

$$
B_{\mu} z_{\lambda}+A \circ g^{-1}\left(J_{\mu}^{B(\cdot, \lambda)} z_{\lambda}, \lambda\right) \text { with } g\left(x_{\lambda}, \lambda\right)=J_{\mu}^{B(\cdot, \lambda)} z_{\lambda} .
$$

(ii) We have assumed that (2.3) has a unique solution $\bar{x}$. By Lemma 2.1 above, we deduce that problem (2.4) admits a solution $\bar{z}$, for $\bar{\lambda} \in \Lambda$.

Now let $\vartheta$ be a closed convex neighborhood of $\bar{z}$. We will use Lemma 2.1 above to study the sensitivity of variational inclusions. More precisely, we want to investigate those conditions under which, for each $z_{\lambda}$ near $\bar{z}$ (respectively $x_{\lambda}$ near $\bar{x}$ ), the function $z_{\lambda}:=z(\lambda)$ (respectively $x_{\lambda}:=x(\lambda)$ ) is continuous or Lipschitz continuous.

Definition 1: Let $A$ be an operator defined on $\vartheta \times \Lambda$. Then, for all $x, y \in \vartheta$, the operator is said to be

(i) locally strongly monotone if there exists a constant $\alpha>0$ such that

$$
\langle A(x, \lambda)-A(y, \lambda), x-y\rangle \geq \alpha\|x-y\|^{2},
$$

(ii) locally Lipschitz continuous if there exists a constant $\beta>0$ such that

$$
\|A(x, \lambda)-A(y, \lambda)\| \leq \beta\|x-y\| \text {. }
$$

It is clear that $\alpha \leq \beta$.

\section{The Main Results}

We consider the case when the solutions of the parametric Wiener-Hopf equation (2.4) lie in the interior of $\vartheta$. Following the ideas of Dafermos [5] and Noor [12], we consider the map

$$
F(z, \lambda)=J_{\mu}^{B} \mid \vartheta^{(\cdot, \lambda)} z_{\lambda}-\mu A\left(\left(x_{\lambda}, \lambda\right)\right)
$$

where $g\left(x_{\lambda}, \lambda\right)=J_{\mu}^{B} \mid \vartheta^{(\cdot, \lambda)} \quad \begin{aligned} & =g\left(x_{\lambda}, \lambda\right)-\mu A\left(x_{\lambda}, \lambda\right) \\ & z_{\lambda} \text { and } B_{\mid \vartheta}: \operatorname{dom} B \cap \vartheta \rightarrow X\end{aligned}$

We have to show that the map $z \rightarrow F(z, \lambda)$ has a fixed point, which is also a solution of (2.4). First of all, we prove that the map is a contraction with respect to $z$, uniformly in $\lambda \in \Lambda$.

Lemma 3.1: Let the operator $A(\cdot, \lambda)$ be locally strongly monotone with constants $\alpha$, locally Lipschitz continuous with constant $\beta$, and $g(\cdot, \lambda)$ be locally strongly mono- 
tone with constant $\delta$ and locally Lipschitz continuous with constant $\sigma$. If

$$
1-k>0, \alpha>2 \beta \sqrt{k(1-k)} \text { and }\left|\mu-\frac{\alpha}{\beta^{2}}\right|<\frac{\sqrt{\alpha^{2}-4 k(1-k) \beta^{2}}}{\beta^{2}},
$$

then, for all $z_{1}, z_{2} \in \vartheta$ and $\lambda \in \Lambda$, we have:

$$
\left\|F\left(z_{1}, \lambda\right)-F\left(z_{2}, \lambda\right)\right\| \leq \theta\left\|z_{1}-z_{2}\right\|
$$

where

and

$$
k:=\sqrt{1-2 \delta}
$$

$$
\theta:=\frac{k+\sqrt{1-2 \mu \alpha+\mu^{2} \beta^{2}}}{1-k}
$$

Proof: For all $z_{1}, z_{2} \in \vartheta, \lambda \in \Lambda$, by (3.1) and by the triangular inequality, we get

$$
\begin{gathered}
\left\|F\left(z_{1}, \lambda\right)-F\left(z_{2}, \lambda\right)\right\| \leq\left\|x_{1}-x_{2}-\left(g\left(x_{1}, \lambda\right)-g\left(x_{2}, \lambda\right)\right)\right\| \\
+\left\|x_{1}-x_{2}-\mu\left(A\left(x_{1}, \lambda\right)-A\left(x_{2}, \lambda\right)\right)\right\| .
\end{gathered}
$$

Setting $E=\left\|x_{1}-x_{2}-\left(g\left(x_{1}, \lambda\right)-g\left(x_{2}, \lambda\right)\right)\right\|^{2}$, since $g(\cdot, \lambda)$ is strongly monotone and Lipschitz continuous, it follows that:

Similarly,

$$
\begin{gathered}
E=\left\|x_{1}-x_{2}\right\|^{2}-2\left\langle g\left(x_{1}, \lambda\right)-g\left(x_{2}, \lambda\right), x_{1}-x_{2}\right\rangle \\
+\left\|g\left(x_{1}, \lambda\right)-g\left(x_{2}, \lambda\right)\right\|^{2} \leq\left(1-2 \delta+\sigma^{2}\right)\left\|x_{1}-x_{2}\right\|^{2} .
\end{gathered}
$$

$$
\left\|x_{1}-x_{2}-\mu\left(T\left(x_{1}\right)-T\left(x_{2}\right)\right)\right\|^{2} \leq\left(1-2 \mu \alpha+\mu^{2} \beta^{2}\right)\left\|x_{1}-x_{2}\right\|^{2} .
$$

From (3.5), (3.6) and (3.7), we obtain:

$$
\left\|F\left(z_{1}, \lambda\right)-F\left(z_{2}, \lambda\right)\right\| \leq\left(\sqrt{1-2 \delta+\sigma^{2}}+\sqrt{1-2 \mu \alpha+\mu^{2} \beta^{2}}\right)\left\|x_{1}-x_{2}\right\| .
$$

According to (3.6) and using the nonexpansiveness of the resolvent, we can write:

$$
\begin{gathered}
\left\|x_{1}-x_{2}\right\| \leq\left\|x_{1}-x_{2}-\left(g\left(x_{1}, \lambda\right)-g\left(x_{2}, \lambda\right)\right)+J_{\mu}^{B}\left|\vartheta^{(\cdot, \lambda)} z_{1}-J_{\mu}^{B}\right| \vartheta^{(\cdot, \lambda)} z_{2}\right\| \\
\leq k\left\|x_{1}-x_{2}\right\|+\left\|z_{1}-z_{2}\right\|
\end{gathered}
$$

thus,

$$
\left\|x_{1}-x_{2}\right\| \leq \frac{1}{1-k}\left\|z_{1}-z_{2}\right\|
$$

which combined with (3.8), yields:

$$
\left\|F\left(z_{1}, \lambda\right)-F\left(z_{2}, \lambda\right)\right\| \leq \theta\left\|z_{1}-z_{2}\right\| .
$$

Since $\theta<1$ for $\mu$ satisfying (3.2), it follows that the map $z \rightarrow F(z, \lambda)$ is a contraction and has a fixed point $z(\lambda)$, the solution of the parametric Wiener-Hopf equations 
(2.4).

Remark 3.1: Since $\bar{z}$ is a solution of (2.4) for $\lambda=\bar{\lambda}$, it is then easy to show that $\bar{z}$ is the unique fixed point in $\vartheta$ of the map $F(\cdot, \bar{\lambda})$. In other words,

$$
\bar{z}=z(\bar{\lambda})=F(z(\bar{\lambda}), \bar{\lambda})
$$

Using Lemma 3.1, we prove the continuity of the solution $z(\lambda)$ (respectively, $x(\lambda)$ ) of (2.4) (respectively (2.3)) which is the main motivation of the next result.

Lemma 3.2: If the operators $A(x, \cdot)$ and $g(\cdot, \lambda)$ are continuous (or Lipschitz continuous), then the functions $z(\lambda)$ is continuous (or Lipschitz continuous) at $\lambda=\bar{\lambda}$. If in addition, the map $\lambda \rightarrow J_{\mu}^{B} \mid \vartheta^{(\cdot, \lambda)} \bar{z}$ is continuous (or Lipschitz_continuous), the function $x(\lambda)$ is in turn continuous (or Lipschitz continuous) at $\lambda=\bar{\lambda}$.

Proof: For $\lambda \in \Lambda$, using Lemma 3.1 and the triangular inequality, we have:

$$
\begin{gathered}
\|z(\lambda)-z(\bar{\lambda})\|=\|F(z(\lambda), \lambda)-F(\bar{z}, \bar{\lambda})\| \\
\leq\|F(z(\lambda), \lambda)-F(\bar{z}, \lambda)\|+\|F(\bar{z}, \lambda)-F(\bar{z}, \bar{\lambda})\| \\
\leq \theta\|z(\lambda)-\bar{z}\|+\|F(\bar{z}, \lambda)-F(\bar{z}, \bar{\lambda})\| .
\end{gathered}
$$

On the other hand, from (3.1):

$$
\|F(\bar{z}, \lambda)-F(\bar{z}, \bar{\lambda})\|=\|g(\bar{x}, \lambda)-g(\bar{x}, \lambda)-\mu(A(\bar{x}, \lambda)-A(\bar{x}, \bar{\lambda}))\| .
$$

Combining (3.10) and (3.11), we obtain:

$$
\|z(\lambda)-\bar{z}\| \leq \frac{1}{1-\theta}(\mu\|A(\bar{x}, \lambda)-A(\bar{x}, \bar{\lambda})\|+\|g(\bar{x}, \lambda)-g(\bar{x}, \bar{\lambda})\|),
$$

from which the first part of the desired result follows.

Now, we have:

$$
\begin{gathered}
\|x(\lambda)-x(\bar{\lambda})\| \leq\|x(\lambda)-\bar{x}-(g(x(\lambda), \lambda)-g(\bar{x}, \lambda))\|+\| g(x(\lambda, \lambda)-g(\bar{x}, \lambda) \| \\
\leq k\|x(\lambda)-\bar{x}\|+\|g(x(\lambda), \lambda)-g(\bar{x}, \bar{\lambda})\|+\|g(\bar{x}, \bar{\lambda})-g(\bar{x}, \lambda)\| .
\end{gathered}
$$

Since $g(x(\lambda))=J_{\mu}^{B} \mid \vartheta^{(\cdot, \lambda)} z(\lambda)$ and $g(\bar{x})=g(x(\bar{\lambda}))=J_{\mu}^{B} \mid \vartheta^{(\cdot, \bar{\lambda})} \bar{z}$, we can write:

$$
\begin{gathered}
\|x(\lambda)-x(\bar{\lambda})\| \leq \frac{1}{1-k}\left(\|z(\lambda)-\bar{z}\|+\left\|J_{\mu}^{B}\left|\vartheta^{(\cdot, \lambda)} \bar{z}-J_{\mu}^{B}\right| \vartheta^{(\cdot, \bar{\lambda})} \bar{z}\right\|\right. \\
+\|g(\bar{x}, \lambda)-g(\bar{x}, \bar{\lambda})\|) .
\end{gathered}
$$

This, combined with (3.12), yields:

$$
\begin{gathered}
\|x(\lambda)-x(\bar{\lambda})\| \leq \frac{1}{1-k}\left(\frac{\mu}{1-\theta}\|A(\bar{x}, \lambda)-A(\bar{x}, \bar{\lambda})\|+\frac{2-\theta}{1-\theta}\|g(\bar{x}, \lambda)-g(\bar{x}, \bar{\lambda})\|\right) \\
\quad+\frac{1}{1-k}\left\|J_{\mu}^{B}\left|\vartheta^{(\cdot, \lambda)} \bar{z}-J_{\mu}^{B}\right| \vartheta^{(\cdot, \bar{\lambda})} \bar{z}\right\|
\end{gathered}
$$


from which we obtain the required result.

Lemma 3.3: If the assumptions of Lemma 3.2 hold true, then there exists a neighborhood $\aleph \subset \Lambda$ of $\bar{\lambda}$ such that for all $\lambda \in \aleph, z(\lambda)$ (respectively, $x(\lambda)$ ) is the unique solution of (2.3) (respectively, (2.4)) in the interior of $\vartheta$.

Proof: Similar to Lemma 2.5 in Dafermos [5].

We now state and prove the main result of this paper.

Theorem 3.1: Let $\bar{x}$ be the solution of parametric variational inclusions (2.3) and $\bar{z}$ the solution of the parametric Wiener-Hopf equations $(2.4)$ for $\lambda=\bar{\lambda}$. Let $A(\cdot, \lambda)$ and $g(\cdot, \lambda)$ be locally strongly monotone and locally Lipschitz continuous operators on $\vartheta$. If the operators $A(\bar{x}, \cdot)$ and $g(\bar{x}, \cdot)$ are continuous (or Lipschitz continuous) at $\lambda=\bar{\lambda}$, then there exists a neighborhood $\aleph \subset \Lambda$ of $\bar{\lambda}$ such that for $\lambda \in \aleph,(2.4)$ has a unique solution $z(\lambda)$ in the interior of $\vartheta, z(\bar{\lambda})=\bar{z}$, and $z(\lambda)$ is continuous (or Lipschitz continuous) at $\lambda=\bar{\lambda}$. If in addition, the map $\lambda \rightarrow J_{\mu}^{B} \mid \vartheta^{(\cdot, \lambda)} \bar{z}$ is continuous (or Lipschitz continuous) at $\lambda=\bar{\lambda}$, then for $\lambda \in \aleph$ the parametric problem (2.3) has a unique solution $x(\lambda)$ in the interior of $\vartheta, x(\bar{\lambda})=\bar{x}$, and $x(\lambda)$ is continuous (or Lipschitz continuous) at $\lambda=\bar{\lambda}$.

Proof: The proof of this theorem follows from Lemmas 3.1-3.3 and Remark 3.1.

Remark 3.2: It is better to impose assumptions on the operator $B_{\mid \vartheta}(\cdot, \lambda)$, which would imply the continuity or the Lipschitz continuity of the map: $\lambda \rightarrow J_{\mu}^{B} \mid \vartheta^{(\cdot \lambda)} \bar{z}$. It is well known (Brézis [4]) that the graph convergence of the filtered sequence $\left\{B_{\mid \vartheta}(\cdot, \lambda) \mid \lambda \downarrow \bar{\lambda}\right\}$ to $B_{\mid \vartheta}(\cdot, \bar{\lambda})$ implies the pointwise convergence of $\left\{J_{\mu}^{B}\left|\vartheta^{(\cdot, \lambda)} z\right| \lambda \downarrow \bar{\lambda}\right\}$ to $J_{\mu}^{B} \mid \theta^{(\cdot, \bar{\lambda}) z}$ for all $\mu>0$ and for all $z \in X$.

To have the Lipschitz continuity, we introduce a localization of the Hausdorff metric and consider a pseudo-Lipschitz property introduced by Aubin [3].

Definition 3.1: A subset $C(\lambda) \rightarrow 2^{x}$ is said to be pseudo-Lipschitz at $(\bar{\lambda}, \bar{x})$ if there exist a neighborhood $W$ of $\bar{\lambda}$, a neighborhood $\Im$ of $\bar{x}$, and a constant $\xi>0$ such that:

$$
C(\lambda) \cap \Im \subset C\left(\lambda^{\prime}\right)+\xi\left|\lambda-\lambda^{\prime}\right| b(0,1) \quad \forall \lambda, \lambda^{\prime} \in \Lambda \cap W
$$

with $b(0,1)$ denoting the closed unit ball of $X$.

Now, let $C$ and $D$ be two subsets of $X$ and $x \in C \cap D$. For any neighborhood $\Im$ of $x$, we define the localized Hausdorff metric between $C$ and $D$ with respect to $\Im$ by:

$$
\operatorname{Haus}_{\Im}(C, D)=\max (\mathrm{e}(C \cap \Im, D) ; \mathrm{e}(D \cap \Im, C))
$$

where $\mathrm{e}(C, D)$ is the excess of $C$ on $D$, and is defined by:

$$
\mathrm{e}(C, D)=\sup _{x \in C} \operatorname{dist}(x, D), \text { with } \operatorname{dist}(x, D)=\inf _{y \in D}\|x-y\| .
$$

In view of Definition 3.1, we easily conclude that the pseudo-Lipschitz of $C(\lambda)$ at $(\bar{\lambda}, \bar{x})$ can be rewritten as:

$$
\operatorname{Haus}_{\Im}\left(C(\lambda), C\left(\lambda^{\prime}\right)\right) \leq \xi\left|\lambda-\lambda^{\prime}\right| \quad \forall \lambda, \lambda^{\prime} \in \Lambda \cap W .
$$

The next results contains a fundamental estimate from which we will derive Lipschitz properties of solutions. 
Proposition 3.1: Let $z \in \vartheta$. The following estimate holds true:

$$
\left\|J_{\mu}^{B}\left|\vartheta^{(\cdot, \lambda)} z-J_{\mu}^{B}\right| \vartheta^{(\cdot, \bar{\lambda})} z\right\| \leq(2+\mu) \operatorname{Haus}_{\Im}\left(B_{\mid \vartheta}(\cdot, \lambda), B_{\mid \vartheta}(\cdot, \bar{\lambda})\right)
$$

with $\Im=\max \left(1, \frac{2}{\mu}\right) \vartheta \times \vartheta$, and $\left.B\right|_{\vartheta}$ is identified by its graph.

Proof: Suppose that $\operatorname{Haus}_{\Im}\left(B_{\mid \vartheta}(\cdot, \lambda), B_{\mid \vartheta}(\cdot, \bar{\lambda})<\eta\right.$, for some $\eta>0$.

Because $\left(B_{\mid \vartheta}(\cdot, \lambda)\right)_{\mu} z \in B_{\mid \vartheta}(\cdot, \lambda)\left(J_{\mu}^{B} \mid \vartheta(\cdot, \lambda) z\right)$ and by the definition of the Yosida approximate, we get:

$$
\left(J_{\mu}^{B} \mid \vartheta^{(\cdot, \lambda)} z,\left(B_{\mid \vartheta}\right)_{\mu} z\right) \in B_{\mid \vartheta}(\cdot, \lambda) \cap \max \left(1, \frac{2}{\mu}\right) \vartheta \times \vartheta
$$

By definition of the localized Hausdorff metric, there exists $\left(z^{\prime}, y^{\prime}\right) \in B_{\mid \vartheta}(\cdot, \bar{\lambda})$ such that:

$$
\left\|z^{\prime}-J_{\mu}^{B} \mid \vartheta^{(\cdot, \lambda)} z\right\| \leq \eta \text { and }\left\|y^{\prime}-\left(B_{\mid \vartheta}(\cdot, \lambda)\right)_{\mu} z\right\| \leq \eta
$$

Set $z_{\mu}=z^{\prime}+\mu y^{\prime}$, which implies $z^{\prime}=J_{\mu}^{B} \mid \vartheta^{(\cdot, \bar{\lambda})} z_{\mu} ;$ thus,

and

$$
\left\|J_{\mu}^{B}\left|\vartheta^{(\cdot, \bar{\lambda})} z_{\mu}-J_{\mu}^{B}\right| \vartheta^{(\cdot, \lambda)} z\right\| \leq \eta
$$

$$
\begin{gathered}
\left\|J_{\mu}^{B}\left|\vartheta^{(\cdot, \lambda)} z-J_{\mu}^{B}\right| \vartheta^{(\cdot, \bar{\lambda})} z\right\| \leq\left\|J_{\mu}^{B}\left|\vartheta^{(\cdot, \lambda)} z-J_{\mu}^{B}\right| \vartheta^{(\cdot, \bar{\lambda})} z_{\mu}\right\| \\
+\left\|J_{\mu}^{B}\left|\vartheta^{(\cdot, \bar{\lambda})} z_{\mu}-J_{\mu}^{B}\right| \vartheta^{(\cdot, \bar{\lambda})} z\right\| \leq \eta+\left\|z_{\mu}-z\right\| .
\end{gathered}
$$

On the other hand,

$$
\begin{aligned}
\left\|z_{\mu}-z\right\|=\left\|z^{\prime}-z+\mu y^{\prime}\right\|= & \left\|z^{\prime}-J_{\mu}^{B} \mid \vartheta^{(\cdot, \lambda)} z+\mu\left(y^{\prime}-\left(B_{\mid \vartheta}(\cdot, \lambda)\right)_{\mu} z\right)\right\| \\
& \leq(1+\mu) \eta .
\end{aligned}
$$

Hence,

$$
\left\|J_{\mu}^{B}\left|\vartheta^{(\cdot, \lambda)} z-J_{\mu}^{B}\right| \vartheta^{(\cdot, \bar{\lambda})} z\right\| \leq(2+\mu) \eta
$$

from which the result follows by letting $\eta$ tend to $\operatorname{Haus}_{\Im}\left(B_{\mid \vartheta}(\cdot, \lambda), B{ }_{\mid \vartheta}(\cdot, \bar{\lambda})\right)$.

Due to Lemma 3.2 and Proposition 3.1, we obtain the following result:

Proposition 3.2: If the operators $A(\bar{x}, \cdot)$ is Lipschitz continuous with constant $\gamma$, $g(\bar{x}, \cdot)$ is Lipschitz continuous with constant $\tau$, and there exists $\bar{y} \in B(\bar{x})$ such that $B$ is pseudo-Lipschitz at $(\bar{\lambda},(\bar{x}, \bar{y}))$; then:

and

$$
\|z(\lambda)-\bar{z}\| \leq \frac{1}{1-\theta}(\mu \gamma+\tau)|\lambda-\bar{\lambda}|
$$

$$
\|x(\lambda)-x(\bar{\lambda})\| \leq \frac{1}{1-k}\left(\frac{(2-\theta) \tau+\gamma \mu}{1-\theta}+(2+\mu) \xi\right) \mid \lambda-\bar{\lambda}
$$


Remark 3.3: In the special case where $B(\cdot, \lambda):=N_{k_{\lambda}}$, the normal cone to a closed convex set $K_{\lambda}$ and $g(\cdot, \lambda)=I,(2.3)$ reduces to

$$
\text { find } x_{\lambda} \in X ;\left(A\left(x_{\lambda}, \lambda\right), y-x_{\lambda}\right) \geq 0 \text { for all } y \in K_{\lambda}
$$

and we recover the main result of Noor [12]. Now suppose $C_{\lambda}$ is defined by the following system of linear equalities and inequalities

$$
K(\lambda)=\left\{x \in \mathbb{R}^{n}, c x=\lambda_{1}, D x \leq \lambda_{2}\right\}
$$

where $\lambda=\left(\lambda_{1}, \lambda_{2}\right) \in \mathbb{R}^{p} \times \mathbb{R}^{q}$, and $C, D$ are $p \times n$ and $q \times n$ real matrices, then, from a result in Yen [22], there exists $k>0$ such that

$$
K\left(\lambda^{\prime}\right) \subset K(\lambda)+k\left|\lambda-\lambda^{\prime}\right| b(0,1) \quad \forall \lambda, \lambda^{\prime} \in \Lambda=\left\{\kappa \in \mathbb{R}^{\tau} ; K(\lambda) \neq 0\right\} .
$$

If $K(\lambda)$ is given by the following formula

$$
D(\lambda)=\left\{x \in \mathbb{R}^{n} \mid x \in C, g_{i}(x, \lambda) \leq 0, \quad i=1, \ldots, p, g_{i}(x, \lambda)=0, i=p+1, \ldots, q\right\},
$$

where $C$ is a closed subset and $g_{i}: X \times \Lambda \rightarrow \mathbb{R}, i=1, \ldots, q$ are locally Lipschitz functions. It was proved in [3] that the set valued map $K: \Lambda \rightarrow 2^{\mathbb{R}^{n}}$ is pseudoLipschitz at $(\bar{x}, \bar{\lambda})$ if a certain qualification condition holds true. More precisely, assume that the following condition is satisfied

$$
\left.\begin{array}{c}
\theta=\left(\theta_{1}, \ldots, \theta_{q}\right) \in \mathbb{R}^{q} \\
\theta_{i} \geq 0 \text { and } \theta_{i} g_{i}(\bar{x}, \bar{\lambda})=0, i=1, \ldots, p \\
0 \in \sum_{i=1}^{n} \theta_{i} \pi_{1}\left(\partial g_{i}(\bar{x}, \bar{\lambda})\right)+N_{c}(\bar{x})
\end{array}\right\} \Rightarrow \theta=0
$$

where $N_{c}(\bar{x})$ is the Clarke normal cone to $C$ at $\bar{x}, \partial g_{i}(\bar{x}, \bar{\lambda})$ is the Clarke generalized gradient of $g$ at $(\bar{x}, \bar{\lambda})$ and

$$
\pi_{1}\left(\partial g_{i}(\bar{x}, \bar{\lambda})\right)=\left\{x^{*} \in \mathbb{R}^{n}: \exists \lambda^{*} \in \mathbb{R}^{r},\left(x^{*}, \lambda^{*}\right) \in \partial g_{i}(\bar{x}, \bar{\eta})\right\}
$$

then $K$ is pseudo-Lipschitz at $(\bar{x}, \bar{\lambda})$.

\section{References}

[1] Attouch, H., Moudafi, A. and Riahi, H., Quantitative stability analysis for maximal monotone operators and semigroups of contractions, Nonl. Anal. 21:9 (1993), 695-723.

[2] Attouch, H. and Théra, M., A general duality principle for the sum of two maximal monotone operators, J. Convex Anal. 3 (1997), 1-24.

[3] Aubin, J.P., Lipschitz behavior of solutions to convex minimization problems, Math. Oper. Res. 9 (1984), 87-111. 
[4] Brézis, H., Opérateurs Maximaux Montones et Sem-groupes de Contractions dans les espaces de Hilbert, North-Holland, Amsterdam 1973.

[5] Dafermos, S., Sensitivity analysis in variational inequalities, Math. Oper. Res. 13 (1988), 421-434.

[6] Fiacco, A.V., Introduction to Sensitivity and Stability Analysis in Nonlinear Programming, Academic Press, New York 1983.

[7] Giannessi, F. and Mangeri, A., Variational Inequalities and Network Equilibrium Problems, Plenum Press, New York 1995.

[8] Janin, R. and Gauvin, J., Lipschitz type stability in nonsmooth convex programs, SIAM Optimization, to appear.

[9] Kyparisis, J., Sensitivity analysis framework for variational inequalities, Math. Prog. 38 (1987), 203-213.

[10] Kyparisis, J., Sensitivity analysis for variational inequalities and complementarity problems, Ann. Opers. Res. 27 (1990), 143-174.

[11] Noor, A.M., General algorithm and sensitivity analysis for variational inequalities, J. Appl. Math. Stoch. Anal. 5 (1992), 29-42.

[12] Noor, A.M., Sensitivity analysis for variational inequalities, Optimization 41 (1997), 207-217.

[13] Noor, M.A., Numerical methods for monotone mixed variational inequalities, Adv. Nonl. Var. Ineq. 1 (1998), 51-79.

[14] Noor, M.A., Some recent advances in variational inequalities, Part I: Basic concepts, New Zealand J. Math. 26 (1997), 53-80.

[15] Noor, M.A., Some recent advances in variational inequalities, Part II: Other concepts, New Zealand J. Math. 26 (1997), 229-255.

[16] Pang, J.S. and Ralph, D., Piecewise smoothness, local invertibility and parametric analysis of normal maps, Math. Oper. Res. 21 (1996), 401-426.

[17] Qui, Y. and Magnanti, T.L., Sensitivity analysis for variational inequalities defined on polyhedral sets, Math. Oper. Res. 14 (1989), 410-432.

[18] Robinson, S.M., Stability theory for systems of inequalities, Part II: Differentiable nonlinear systems, SIAM J. Numer. Anal. 13:4 (1975), 754-769.

[19] Robinson, S.M., Sensitivity analysis of variational inequalities by normal map techniques, In: Variational Inequalities and Network Equilibrium Problems (ed. by F. Giannessi and Maugeri), Plenum Press, New York 1995.

[20] Tobin, R.L., Sensitivity analysis for variational inequalities, J. Optim. Theory Appl. 48 (1986), 191-204.

[21] Yen, N.D., Lipschitz continuity of solutions of variational inequalities with parametric polyhedral constraint, Math. Oper. Res. 20 (1995), 695-708.

[22] Yen, N.D., Holder continuity of solutions to parametric variational inequality, Appl. Math. Optim. 31 (1995), 245-255. 


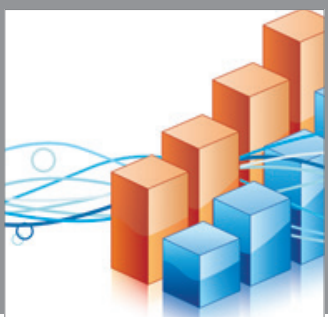

Advances in

Operations Research

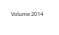

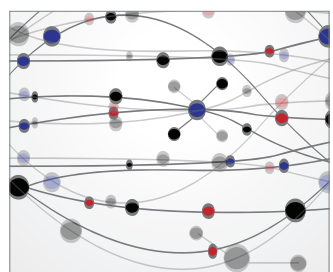

\section{The Scientific} World Journal
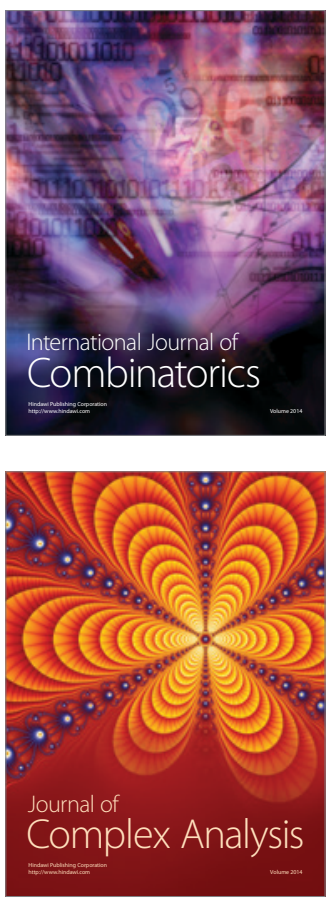

International Journal of

Mathematics and

Mathematical

Sciences
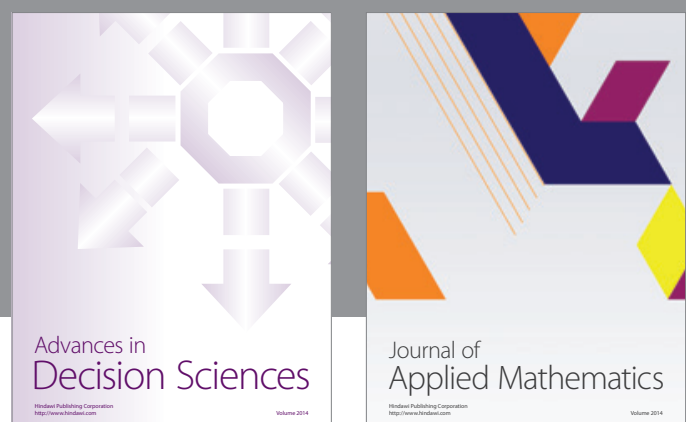

Journal of

Applied Mathematics
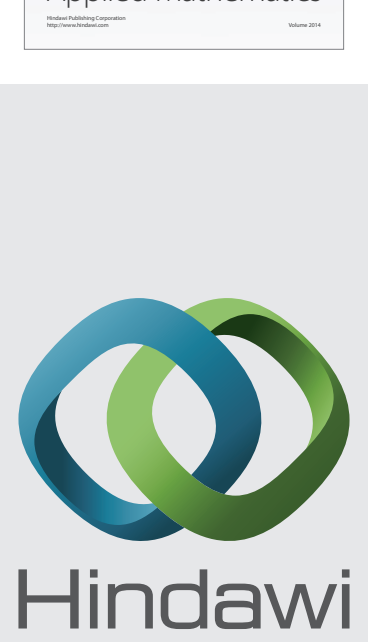

Submit your manuscripts at http://www.hindawi.com
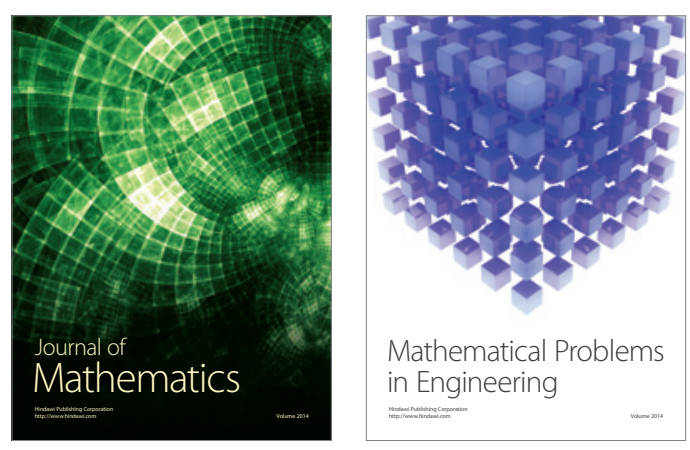

Mathematical Problems in Engineering
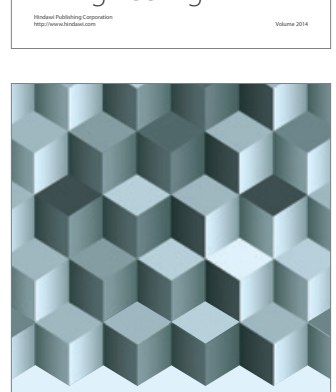

Journal of

Function Spaces
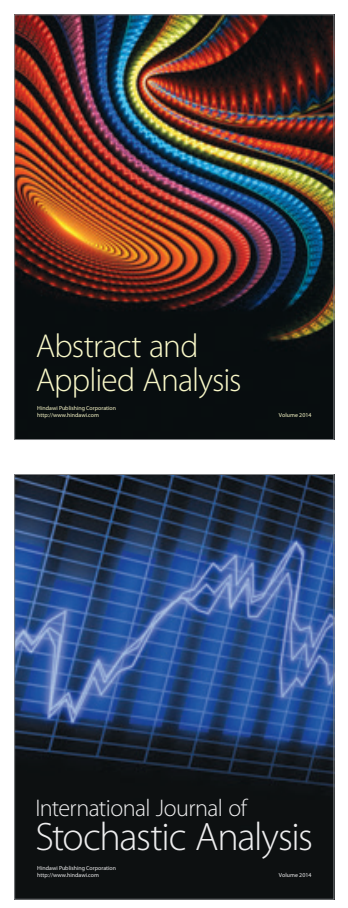

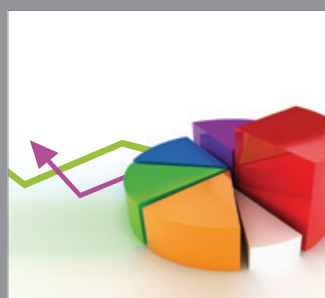

ournal of

Probability and Statistics

Promensencen
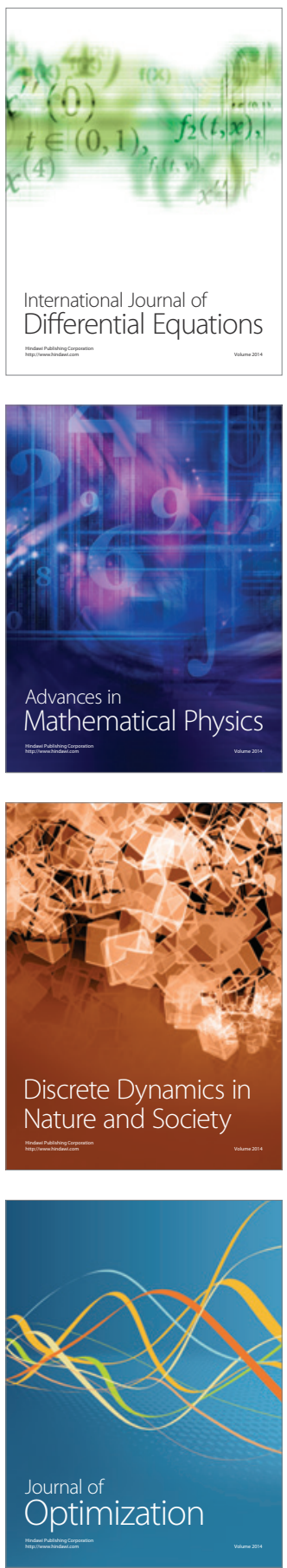